\title{
Some good-filtration subgroups of simple algebraic groups
}

\author{
Chuck Hague* \\ Department of Mathematical Sciences \\ University of Delaware \\ 501 Ewing Hall \\ Newark, DE 19716 \\ George McNinch ${ }^{1}$ \\ Department of Mathematics \\ Tufts University \\ 503 Boston Ave \\ Medford, MA 02155
}

\begin{abstract}
Let $G$ be a connected and reductive algebraic group over an algebraically closed field of characteristic $p>0$. An interesting class of representations of $G$ consists of those $G$-modules having a good filtration - i.e. a filtration whose layers are the induced highest weight modules obtained as the space of global sections of $G$-linearized line bundles on the flag variety of $G$. Let $H \subset G$ be a connected and reductive subgroup of $G$. One says that $(G, H)$ is a Donkin pair, or that $H$ is a good filtration subgroup of $G$, if whenever the $G$-module $V$ has a good filtration, the $H$-module $r e s_{H}^{G} V$ has a good filtration.

In this paper, we show when $G$ is a "classical group" that the optimal $S L_{2}$-subgroups of $G$ are good filtration subgroups. We also consider the cases of subsystem subgroups in all types and determine some primes for which they are good filtration subgroups.
\end{abstract}

Keywords: algebraic groups, Donkin pairs, good filtrations

\section{Contents}

$\begin{array}{lll}1 & \text { Introduction } & 1\end{array}$

2 Preliminaries 2

3 The groups of interest $\quad 7$

4 Main results 13

\section{Introduction}

Let $G$ be a connected and reductive group over an algebraically closed field of characteristic $p>0$, and let $H \subset G$ be a closed subgroup which is also connected and reductive. We are concerned

\footnotetext{
*Corresponding author

Email addresses: hague emath. udel .edu (Chuck Hague), george.mcninch@tufts .edu (George McNinch)

URL: http://sites.google.com/site/chuckhague/(Chuck Hague), http://gmeninch.math.tufts.edu/ (George McNinch)

${ }^{1}$ Research of McNinch supported in part by the US NSA award H9230-11-1-0164.
} 
here with the linear representations of the algebraic group $G$-i.e. with $G$-modules - and with their restriction to $H$.

Of particular interest are the induced G-modules $\nabla_{G}(\lambda)$ and the induced $H$-modules $\nabla_{H}(\lambda)$ obtained as global sections of equivariant bundles on the associated flag varieties; see $\$ 2.1$. One says that $H$ is a good-filtration subgroup of $G$ - or that $(G, H)$ is a Donkin pair - provided that for any induced G-module $V$ the $H$-module $r e s_{H}^{G} V$ obtained from $V$ by restriction to $H$ has an exhaustive filtration whose successive quotients are induced $H$-modules.

Donkin proved in [7] - under some mild assumptions on the characteristic - that a Levi factor of a parabolic subgroup of $G$ is always a good-filtration subgroup; subsequently, Mathieu gave an unconditional proof [14] of this result using the geometric method of Frobenius splitting (cf. also the accounts in [3] $\$ 4,[11]$ Ch. G, and [22]).

In [4], Brundan proved that a large class of reductive spherical subgroups of $G$ are good filtration subgroups, under mild restrictions on $p$; recall that a subgroup $H$ is said to spherical if there is a dense $H$-orbit on the flag variety $G / B$ of $G$. In that paper, Brudan also conjectured that $H$ is a good filtration subgroup if either (i) $H$ is the centralizer of a graph automorphism of $G$, or (ii) $H$ is the centralizer of an involution of $G$ and $p>2$. Brundan's conjecture is now a theorem; many cases were covered already in [4] and the remaining cases were handled by van der Kallen in [21].

In this paper we extend the study of Donkin pairs to more reductive subgroups of $G$. In particular, we consider two classes of reductive subgroups: optimal $S L_{2}$-subgroups and the so-called subsystem subgroups. In $₫ 2$ we give preliminaries on algebraic groups, good filtrations, and optimal $S L_{2}$ subgroups.

In this paper a group of classical type, or just a classical group, will be a group isomorphic to $S L(V)$ or the stabilizer of a nondegenerate alternating or bilinear form $\beta$ when $p>2$. In 3 we give a general criterion for a reductive subgroup of a group of classical type to be a good filtration subgroup (3.2.6). Since a group of classical type is not simply-connected when the form $\beta$ is symmetric we also consider the simply-connected covers of these groups (3.2.7). We then give a criterion for checking when a reductive subgroup of a group of exceptional type is a good filtration subgroup (Theorem 3.6.3).

In $\$ 4$ we give our main results. In $\$ 4.1$ we consider the case in which $G$ is a classical group and $S \subset G$ is an optimal $S L_{2}$ subgroup, a notion essentially due to Seitz [20]; we follow the characterization of these subgroups given in [15]. The main result of this section is Theorem 4.1.2, which states that optimal $S L_{2}$-subgroups of classical groups are good filtration subgroups. Our proof is modeled on arguments of Donkin from [7]. We also consider optimal $S L_{2}$ subgroups of the simply-connected covers of classical groups (Theorem 4.1.4). In $\$ 4.2$ we consider optimal $S L_{2}$ subgroups of exceptional groups. In these theorems we crucially use induction arguments which reduce to the case of a distinguished optimal $S L_{2}$ subgroup.

Recall that a subsystem subgroup of $G$ is a connected semisimple subgroup which is normalized by a maximal torus. In $\$ 4.3$ we consider arbitrary subsystem subgroups of semisimple groups. Since Brundan's conjecture implies that every subsystem subgroup of a group of type $A, B, C$, or $D$ is a good filtration subgroup when $p>2$, we only consider the exceptional case. By the transitivity of the good filtration subgroup property and the fact that Levi factors of parabolic subgroups are good filtration subgroups, it suffices to consider only the case where the subsystem subgroup is of maximal rank (= rank $G$ ). The main result in this section is Theorem 4.3.3, which gives primes $p$ for which the maximal rank reductive subgroups not already covered by the Brundan conjecture are good filtration subgroups.

Also, we would like to thank the anonymous referee for suggesting useful improvements.

\section{Preliminaries}

\subsection{Induced modules for reductive groups}

Let $k$ be an algebraically closed field of positive characteristic $p$ and let $G$ be a connected and reductive algebraic group over $k$. Fix a maximal torus $T \subset G$, and choose a Borel subgroup $B \subset G$ 
containing $T$. For us, a representation of a linear algebraic group always means a rational representation; namely, a co-module for the coordinate algebra.

We write $X^{*}(T)$ for the character group and $X_{*}(T)$ for the co-character group of the torus $T$. We write $(\lambda, \phi) \mapsto\langle\lambda, \phi\rangle \in \mathbf{Z}$ for the natural pairing $X^{*}(T) \times X_{*}(T) \rightarrow \mathbf{Z}$. Recall that the choice of the Borel subgroup $B$ determines a system of positive roots $R^{+}$of the set of roots $R \subset X^{*}(T)$.

Each character $\lambda \in X^{*}(T)$ determines a $G$-linearized line bundle $\mathscr{L}(\lambda)$ on the flag variety $G / B$. The group $G$ acts linearly on the space of global sections

$$
H^{0}(G / B, \mathscr{L}(\lambda)) ;
$$

we write $\nabla_{G}(\lambda)$ for this $G$-module (which is denoted $H_{G}^{0}(\lambda)=H^{0}(\lambda)$ in [11] $\left.\S I I\right)$. Then $\nabla_{G}(\lambda)$ is non- 0 if and only if $\lambda$ is dominant; i.e. if and only if $\left\langle\lambda, \alpha^{\vee}\right\rangle \geq 0$ for each $\alpha \in R^{+}$. The representations $\nabla_{G}(\lambda)$ are known as induced modules for $G$.

Assume that $G$ is quasisimple; in this case, we number the nodes of the Dynkin diagram of $G$ - and hence the simple roots and fundamental dominant weights - as in Bourbaki [2], Plate I-IX. Let $\mathfrak{\omega}_{i} \in X^{*}(G) \otimes \mathbf{Q}$ denote the fundamental dominant weights; if $\alpha_{1}, \ldots, \alpha_{r}$ are the simple roots with corresponding co-roots $\alpha_{i}^{\vee} \in X_{*}(T)$, then $\left\langle\omega_{i}, \alpha_{j}^{\vee}\right\rangle=\delta_{i j}$ for $1 \leq i, j \leq r$. Of course, $G$ is simply connected if and only if $\omega_{i} \in X^{*}(T)$ for $1 \leq i \leq r$.

\subsection{Modules with a good filtration}

Let $V$ be any $G$-module. A collection of $G$-submodules $V_{i} \subset V$ for $i \in \mathbf{Z}_{\geq 0}$ forms a filtration of $V$ provided that $V_{i} \subset V_{i+1}$ for $i \geq 0$ and that $V=\bigcup_{i \geq 0} V_{i}$. The layers of the filtration are the quotient modules $V_{i} / V_{i-1}$.

The filtration of $V$ is said to be a good filtration if for each $i \geq 1$, the layer $V_{i} / V_{i-1}$ is either 0 or is isomorphic to an induced module $\nabla_{G}\left(\lambda_{i}\right)$ for some dominant weight $\lambda_{i}$.

For a $G$-module $V$ with a good filtration, the support of $V$ (written as $\operatorname{Supp}(V)$ ) is the set of $\lambda \in X_{G}^{+}$ for which $\nabla_{G}(\lambda)$ occurs as a layer in a good filtration of $V$. It follows from [11], Prop. II.4.16 that the support of $V$ is independent of the choice of good filtration of $V$.

2.2.1. Let $(*) \quad 0 \rightarrow V \rightarrow E \rightarrow W \rightarrow 0$ be a short exact sequence of $G$-modules.

(a) Assume that $V$ has a good filtration. Then $E$ has a good filtration if and only if $W$ has a good filtration.

(b) If the sequence $(*)$ is split exact, and if $E$ has a good filtration, then both $V$ and $W$ have a good filtration.

Proof. Assertion (a) follows from the "homological" characterization of good filtrations found in [11], Prop. II.4.16, and (b) is an immediate consequence of (a).

We also observe the following:

2.2.2. If the G-module $V$ has a filtration for which each quotient $V_{i} / V_{i-1}$ has a good filtration for $i \geq 1$, then $V$ has a good filtration.

Proof. This is straightforward when $V$ is finite dimensional; the general case is obtained in [7], Prop. 3.1.1.

The following important result was first obtained for $p \gg 0$ by J. Wang, with improvements to the prime $p$ by Donkin [7] (under some small restrictions), and in general by Mathieu [14].

2.2.3 (Wang, Donkin, Mathieu). If $V$ and $W$ are finite dimensional G-modules each having a good filtration, then the G-module $V \otimes W$ has a good filtration.

We also have the following useful fact.

2.2.4. Let $\lambda, \mu \in X_{G}^{+}$. There is a surjective mapping of $G$-modules $\nabla_{G}(\lambda) \otimes \nabla_{G}(\mu) \rightarrow \nabla_{G}(\lambda+\mu)$ whose kernel has a good filtration. 
Proof. It follows from 2.2.3 that $M=\nabla_{G}(\lambda) \otimes \nabla_{G}(\mu)$ has a good filtration. Since $\lambda+\mu$ is the highest weight of $M$ we have $\lambda+\mu \in \operatorname{Supp}(M)$. Since any weight $\gamma$ of $M$ satisfies $\gamma \leq \lambda+\mu$, it follows that for $\sigma \in \operatorname{Supp}(M) \backslash\{\lambda+\mu\}$ we have $\sigma<\lambda+\mu$ and hence $\operatorname{Ext}_{G}^{1}\left(\nabla_{G}(\sigma), \nabla_{G}(\bar{\lambda}+\mu)\right)=0$ [11], Prop. II.6.20.

Since $\nabla_{G}(\lambda+\mu)$ occurs as a layer in a good filtration of $M$, there is a submodule $V \subseteq M$ with a surjection $f: V \rightarrow \nabla_{G}(\lambda+\mu)$. By the above, $\operatorname{Ext}_{G}^{1}\left(M / V, \nabla_{G}(\lambda+\mu)\right)=0$, so $f$ lifts to a surjection $\widehat{f}: M \rightarrow \nabla_{G}(\lambda+\mu)$, and the kernel of $\widehat{f}$ has a filtration with layers $\nabla_{G}(\sigma)$ for $\sigma \in \operatorname{Supp}(M) \backslash\{\lambda+$ $\mu\}$.

2.2.5. Let $V$ be a G-module with a good filtration. Then $V^{\otimes m}$ has a good filtration for all $m \geq 0$. If $m<p$ then

$$
\bigwedge^{m} V \text { and } \operatorname{Sym}^{m} V
$$

each have a good filtration.

Proof. The observation that $V^{\otimes m}$ has a good filtration follows from 2.2.3 If $m<p$, it is sufficient by 2.2.1 to argue that $\Lambda^{m} V$ and $S y m^{m} V$ are direct summands of $V^{\otimes m}$ as G-modules. Although this fact is well-known we give the proof here for completeness. For this, it suffices to observe that there are $G$-linear splittings $\sigma_{1}: \Lambda^{m} V \rightarrow V^{\otimes m}$ and $\sigma_{2}: \operatorname{Sym}^{m} V \rightarrow V^{\otimes m}$ of the natural surjections

$$
\pi_{1}: V^{\otimes m} \rightarrow \bigwedge^{m} V \quad \text { and } \quad \pi_{2}: V^{\otimes m} \rightarrow S y m^{m} V ;
$$

i.e. $\pi_{i} \circ \sigma_{i}=\mathrm{id}$ for $i=1,2$. We define the required $\sigma_{i}$ as follows; for $v_{1}, \ldots, v_{m} \in V$ one sets

$$
\sigma_{1}\left(v_{1} \wedge \cdots \wedge v_{m}\right)=\frac{1}{m !} \sum_{\tau \in S y m_{m}} \operatorname{sgn}(\tau) v_{\tau(1)} \otimes \cdots \otimes v_{\tau(m)}
$$

and

$$
\sigma_{2}\left(v_{1} \cdots v_{m}\right)=\frac{1}{m !} \sum_{\tau \in S y m_{m}} v_{\tau(1)} \otimes \cdots \otimes v_{\tau(m)}
$$

where $\operatorname{Sym}_{m}$ is the symmetric group on $m$ letters, and $\operatorname{sgn}(\tau) \in\{ \pm 1\} \subset k^{\times}$is the sign of the permutation $\tau \in \operatorname{Sym}_{m}$ (note that if $m>1$, then $p>2$ ). We leave to the reader the task of checking that the rules above yield well-defined G-homomorphisms which determine sections $\sigma_{i}$ to the maps $\pi_{i}$ for $i=1,2$.

\subsection{Donkin pairs}

Let $H \subset G$ be a closed subgroup, and suppose that both $H$ and $G$ are connected and reductive. We choose Borel subgroups $B_{H} \subset H$ and $B_{G} \subset G$ with $B_{H} \subset B_{G}$, and we choose maximal tori $T_{H} \subset B_{H}$ and $T_{G} \subset B_{G}$ with $T_{H} \subset T_{G}$.

Write $X_{G}:=X^{*}\left(T_{G}\right)$ for the weight lattice of $T_{G}$, and let $X_{G}^{+} \subset X_{G}$ denote the dominant weights (determined by the choice of Borel subgroup $B_{G}$ ); similarly, write $X_{H}^{+} \subset X_{H}:=X^{*}\left(T_{H}\right)$. For $\lambda \in X_{G}^{+}$ recall that $\nabla_{G}(\lambda)$ is the induced G-module with highest weight $\lambda$. Similarly, for $\mu \in X_{H}^{+}$we write $\nabla_{H}(\mu)$ for the induced $H$-module with highest weight $\mu$.

Definition 2.3.1. One says that $(G, H)$ is a Donkin pair if whenever $V$ is a $G$-module for which $V$ has a good filtration, then res ${ }_{H}^{G} V$ has a good filtration as $H$-module. One also says that $H$ is a good filtration subgroup of $G$.

Let us write $\mathcal{W}(G, H)$ for the set of dominant weights $\lambda \in X_{G}^{+}$for which $\operatorname{res}_{H}^{G} \nabla_{G}(\lambda)$ has a good filtration as $H$-module.

2.3.2. $(G, H)$ is a Donkin pair if and only if $\lambda \in \mathcal{W}(G, H)$ for each $\lambda \in X_{G}^{+}$.

Proof. This follows from 2.2.2 
An important example of Donkin pairs is given by the following result; as for 2.2.3, this result was obtained first by Donkin [7] (under some mild assumptions) and subsquently by Mathieu [14] in general.

2.3.3. If $L \subset G$ is a Levi factor of a parabolic subgroup of $G$, then $(G, L)$ is a Donkin pair.

Remark 2.3.4. One can understand the earlier result 2.2.3 using the notion of Donkin pairs. Namely, if $G$ is a connected and reductive group, let $\Delta: G \rightarrow G \times G$ denote the diagonal embedding. Then 2.2.3 amounts to the assertion that $(G \times G, \Delta(G))$ is a Donkin pair.

2.3.5. Let $G_{1}$ and $G_{2}$ be connected and reductive algebraic groups, and let $G=G_{1} \times G_{2}$.

(a) $\left(G, G_{1}\right)$ and $\left(G, G_{2}\right)$ are Donkin pairs.

(b) If $H \subset G$ is a connected and reductive subgroup, write $H_{i} \subset G_{i}$ for the image $H_{i}=\pi_{i} H \subset G_{i}$ where $\pi_{i}: G \rightarrow G_{i}$ are the projection mappings. If $\left(G_{i}, H_{i}\right)$ is a Donkin pair for $i=1,2$, then $(G, H)$ is a Donkin pair.

Proof. (a) follows from [6], Prop. 1.2(e).

For (b), argue as in [7] (3.4.6) to see that $\left(G=G_{1} \times G_{2}, H_{1} \times H_{2}\right)$ is a Donkin pair, so it suffices to see that $\left(H_{1} \times H_{2}, H\right)$ is a Donkin pair. Now, the inclusion $H \hookrightarrow H_{1} \times H_{2}$ factors as

$$
H \hookrightarrow H \times H \stackrel{q}{\rightarrow} H_{1} \times H_{2},
$$

where the first map is the diagonal inclusion and $q$ is the restriction of the projection $\pi_{1} \times \pi_{2}$ to $H \times H$. Since the pullback by $q$ of an induced module for $H_{1} \times H_{2}$ is an induced module for $H \times H$ (since $q$ is a surjection), the fact that $\left(H_{1} \times H_{2}, H\right)$ is a Donkin pair now follows from 2.2.3 as in Remark 2.3 .4

2.3.6. Let $G$ be a connected and semisimple group, let $H$ be a connected and reductive subgroup of $G$, and let $\pi: G_{s c} \rightarrow G$ be the simply connected covering group. If $\left(G_{s c}, \pi^{-1} H\right)$ is a Donkin pair, then $(G, H)$ is a Donkin pair.

Proof. Argue via [7], 3.4.3.

\subsection{Checking for a Donkin pair using finitely many dominant weights}

Let $G$ be a semisimple group, and fix a system of simple roots $S \subset R \subset X=X^{*}(T)$. We have the following generalization of a result of Donkin found in [7] Prop. 3.5.4, whose proof we have followed closely.

2.4.1. Suppose that $\lambda_{1}, \ldots, \lambda_{r} \in X_{G}^{+}$have the property

$$
\text { (*) } \quad \mu \in X_{G}^{+} \quad \text { if and only if } \mu \in \sum_{i=1}^{r} \mathbf{Z}_{\geq 0} \lambda_{i}
$$

for $\mu \in X$. If $H$ is a reductive subgroup of $G$, then $(G, H)$ is a Donkin pair if and only if $\lambda_{i} \in \mathcal{W}(G, H)$ for $1 \leq i \leq r$.

Proof. This follows from the same technique as in the proof of [7], Prop. 3.5.4. We give a full proof here for completeness. Consider the partial order on $X$ given as follows: $\lambda \succ \mu$ if and only if $\lambda-\mu=$ $\sum_{\alpha \in S} m_{\alpha} \alpha \in X \otimes_{\mathbf{Z}} \mathbf{Q}$ where $m_{\alpha} \in \mathbf{Q}$ and $m_{\alpha} \geq 0$ for all $\alpha$. Note that if $\lambda \geq \mu$ then $\lambda \succ \mu$. It follows from [9], §13, Exerc. 8 that any dominant weight $\lambda$ satisfies $\lambda \succ 0$. In view of the assumption $(*)$, for any $\mu \in X_{G}^{+}$with $\mu \neq 0$ we have $\mu \prec \mu-\lambda_{j}$ for some $1 \leq j \leq r$.

We now give the proof. If $(G, H)$ is a Donkin pair, it is of course immediate from definitions that each $\lambda_{i} \in \mathcal{W}(G, H)$ for $1 \leq i \leq r$. 
We now suppose that $\lambda_{i} \in \mathcal{W}(G, H)$ for $1 \leq i \leq r$, and we must show that $(G, H)$ is a Donkin pair. Suppose on the contrary that $(G, H)$ is not a Donkin pair. Then there is a weight $\mu \in X_{G}^{+}$for which $\mu \notin \mathcal{W}(G, H)$. We may and will suppose that $\mu \in X_{G}^{+}$is minimal with respect to the partial order $\prec$; i.e., we suppose that $\mu \notin \mathcal{W}(G, H)$ and $\lambda \in \mathcal{W}(G, H)$ for all $\lambda \in X_{G}^{+}$with $\lambda \prec \mu$. Since $0 \in \mathcal{W}(G, H)$ we have $\mu \neq 0$. By hypothesis, as noted above, we have $\mu \succ \mu-\lambda_{j} \in X_{G}^{+}$for some $1 \leq j \leq r$. Since $\lambda_{j} \in \mathcal{W}(G, H)$ by assumption, we see that $\mu-\lambda_{j} \neq 0$.

We now consider the $G$-module $T=\nabla_{G}\left(\mu-\lambda_{j}\right) \otimes \nabla_{G}\left(\lambda_{j}\right)$. By the minimality of $\mu$, we have $\mu-\lambda_{j} \in \mathcal{W}(G, H)$. Since we have also $\lambda_{j} \in \mathcal{W}(G, H)$ by assumption, 2.2.3 implies that $T$ has a good filtration as $G$-module and that $\operatorname{res}_{H}^{G} T$ has a good filtration as an $H$-module.

According to 2.2.4, there is an exact sequence of $G$-modules

$$
0 \rightarrow M \rightarrow T \rightarrow \nabla_{G}(\mu) \rightarrow 0
$$

for which $M$ has a good filtration as $G$-module. Moreover, since the dimension of the $\mu$-weight space in $\nabla_{G}\left(\mu-\lambda_{j}\right) \otimes \nabla_{G}\left(\lambda_{j}\right)$ is 1, it follows from [11], Prop. II.4.16 that in any good filtration of $T$, there is precisely one layer isomorphic to $\nabla_{G}(\mu)$. In particular, $\nabla_{G}(\mu)$ does not appear as a layer in the G-module $M$.

Since any weight $\gamma$ of $T$ satisfies $\mu \geq \gamma$ and in particular $\mu \succ \gamma$, it follows that $\operatorname{res}_{H}^{G} M$ has a good filtration as $H$-module; cf. 2.2.2. It now follows from [11], II.4.17 that $\operatorname{res}_{H}^{G} \nabla_{G}(\mu)$ has a good filtration as $H$-module, so that $\mu \in \mathcal{W}(G, H)$, contrary to assumption. This contradiction establishes that $(G, H)$ is a Donkin pair, as required.

Remark 2.4.2. In particular, we obtain the following statement ([7], Prop. 3.5.4): If $G$ is semisimple and simply connected of rank $r$, then the assumption of 2.4.1 holds for the collection of weights $\lambda_{i}=\omega_{i}$ for $1 \leq i \leq$ $r$, where $\omega_{i}$ is the $i$-th fundamental dominant weight.

On the other hand, suppose that $G$ is semisimple, that $\pi: G_{s c} \rightarrow G$ is its simply connected covering group, and that $H \subset G$ is a reductive subgroup. Write $\tilde{H}=\pi^{-1} H \subset G_{s c}$. The formulation 2.4.1 will allow us to check that $(G, H)$ is a Donkin pair in situations when we are unable to determine whether $\left(G_{s c}, \tilde{H}\right)$ is a Donkin pair, cf 3.2.4 3.2.5 and 3.2.6 below.

\subsection{Optimal $S L_{2}$-subgroups}

In this section, let $H$ be a quasisimple group with root system $R$. We want to consider primes which are good for $G$ or equivalently primes which are good for $R$. Recall that bad (=not good) primes are as follows: the prime $p=2$ is bad whenever $R \neq A_{r}, p=3$ is bad if $R=G_{2}, F_{4}, E_{r}$, and $p=5$ is bad if $R=E_{8}$. Finally, the prime $p$ is said to be very good for $R$ if $p$ is good for $R$ and if $R=A_{r}$ then $p$ does not divide $r+1$.

The following result can be deduced as a consequence of Premet's proof of the Bala-Carter Theorem which classifies the nilpotent $G$-orbits in $\operatorname{Lie}(G)$ :

2.5.1. Let $H$ be a quasisimple group and suppose that the characteristic of $k$ is good for $H$. Let $G$ be a reductive group which is isomorphic to a Levi factor of a parabolic subgroup of $H$. If $X \in \operatorname{Lie}(G)$ is nilpotent, then there is a cocharacter $\lambda: \mathbf{G}_{m} \rightarrow G$ such that

(i) for each $t \in k^{\times}, \operatorname{Ad}(\lambda(t)) X=t^{2} X$.

(ii) the image of $\lambda$ is contained in the derived group of $M=C_{G}(S)$ for some maximal torus $S$ of the group $C_{G}(X)$.

If $\lambda, \lambda^{\prime}: \mathbf{G}_{m} \rightarrow G$ are two cocharacters satisfying (i) and (ii), there is a unique element $u \in R_{u}\left(C_{G}(X)\right)$ such that $\lambda^{\prime}(t)=u \lambda(t) u^{-1}$ for each $t \in k^{\times}$; i.e. $\lambda^{\prime}=\operatorname{Int}(u) \circ \lambda$.

Proof. [17], Proposition 18 shows how to deduce the existence of $\lambda$ from results of [19]. For the conjugacy assertion see [15], Prop/Def 21. 
If $X \in \operatorname{Lie}(G)$ is nilpotent, we say that a cocharacter $\lambda$ satisfying (i) and (ii) of 2.5 .1 is associated with $X$.

Let $X_{0}=\left(\begin{array}{ll}0 & 1 \\ 0 & 0\end{array}\right) \in \operatorname{Lie}\left(S L_{2}\right)$. One says that a homomorphism $\phi: S L_{2} \rightarrow G$ is optimal if the cocharacter of $G$ determined by restriction of $\phi$ to the diagonal torus of $S L_{2}$ is associated with the nilpotent element $X=d \phi\left(X_{0}\right)$.

2.5.2. Let $\pi: G_{1} \rightarrow G$ be a central isogeny and let $\phi: S \rightarrow G_{1}$ be a homomorphism of algebraic groups. Then $\phi$ is optimal if and only if $\pi \circ \phi$ is optimal.

Proof. The assertion follows from [17], Lemma 14.

Theorem 2.5.3 ([15], Theorem 44). Let $X \in \operatorname{Lie}(G)$ be nilpotent and suppose that $X^{[p]}=0$. There exists an optimal homomorphism $\phi: S L_{2} \rightarrow G$ with $X=d \phi\left(X_{0}\right)$. If $\phi^{\prime}$ is another optimal homomorphism $S L_{2} \rightarrow G$ with $X=d \phi\left(X_{0}\right)$, then $\phi$ and $\phi^{\prime}$ are conjugate by a unique element of $R_{u} C_{G}(X)$.

The image $S=\operatorname{image}(\phi)$ of an optimal $S L_{2}$-homomorphism will be called an optimal $S L_{2}$-subgroup (although of course it may be that $S \simeq \mathrm{PSL}_{2}$ is the adjoint group).

One says that an optimal $S L_{2}$-subgroup $S$ of $G$ is distinguished just in case a nilpotent element $0 \neq X \in \operatorname{Lie}(S) \subseteq \operatorname{Lie}(G)$ is distinguished.

2.5.4. If $S$ is an optimal $S L_{2}$-subgroup of a quasisimple group $G$, then $S$ is distinguished if and only if a maximal torus of the centralizer $C_{G}(S)$ is trivial.

Proof. First suppose that $S$ is distinguished. Let $0 \neq X \in \operatorname{Lie}(S)$. Since $X$ is distinguished, by definition a maximal torus $T$ of $C_{G}(X)$ is central in $G$; since $G$ is quaisimple, $T=1$. It follows from [15], Cor. 43 that $C_{G}(S) \subset C_{G}(X)$ so indeed a maximal torus of $C_{G}(S)$ is trivial.

Conversely, suppose that a maximal torus of $C_{G}(S)$ is trivial and let $T \subset C_{G}(X)$ be a maximal torus. To show that $X$ is distinguished, we must argue that $T$ is trivial. Write $\lambda$ for the cocharacter of $G$ obtained by the inclusion of the maximal torus of $S$. By the conjugacy of maximal tori in $C_{G}(X)^{0}$, we may suppose that $T$ is centralized by the image of $\lambda$. But then [15], Cor. 43 shows that $T$ centralizes $S$, so indeed $T=1$.

\section{The groups of interest}

\section{1. "Classical" groups}

Let $V$ be a vector space, and let $\beta$ be a non-degenerate bilinear form on $V$. Write

$$
\Omega(V)=\Omega(V, \beta)=\operatorname{Stab}_{G L(V)}(\beta)^{0}
$$

for the identity component of the stabilizer of $\beta$ in $G L(V)$. There are two cases of interest to us:

(a) If $\beta$ is alternating, then $\Omega(V)=S p(V)$ is a symplectic group.

(b) If $\beta$ is symmetric, then $\Omega(V)=S O(V)$ is a special orthogonal group, when $p>2$.

The definition of the special orthogonal group requires more care when $p=2$, and we ignore this issue.

In this paper, a classical group will mean a group of the form

$$
G=S L(V) \text { or } \quad G=\Omega(V, \beta) \text { in case (a) or (b) for } p>2 \text {. }
$$




\subsection{Good filtration subgroups of classical groups}

Let $G$ be a classical group as in 3.1 . We say that $V$ is the natural representation of the classical group $G$. We will often just write $\Omega(V)$ instead of $\Omega(V, \beta)$.

3.2.1. Let $G$ be a classical group with natural representation $V$. Then $V \simeq \nabla_{G}(\lambda)$ for some dominant weight $\lambda$, and $V$ is simple.

Proof. See, for example, [11], $\S I I .2 .16,17,18$. The simplicity of $V$ when $G=\Omega(V, \beta)$ for $\beta$ symmetric depends in general on the assumption that $p \neq 2$.

Recall that the fundamental dominant weights of $G$ are denoted by $\omega_{1}, \ldots, \omega_{r}$ where $r$ is the rank of the semisimple group $G$; in general $\omega_{i} \in X^{*}(T) \otimes \mathbf{Q}$. In what follows, we abbreviate $X:=X^{*}(T)$.

3.2.2. Let $G=S L(V)$, where $\operatorname{dim} V=r+1$. Then $G$ is a simply connected group with root system of type $A_{r}$, and we have

$$
\nabla_{S L(V)}\left(\omega_{i}\right) \simeq \bigwedge^{i} V \quad \text { for } \quad 1 \leq i \leq r .
$$

Proof. [12], Theorem (25.9) identifies the root system of $S L(V)$. Apply e.g. [7](4.1.1) for the assertion about exterior powers.

3.2.3. Assume that $\beta$ is alternating and non-degenerate and that $\operatorname{dim} V=2 r$. Then $\Omega(V)=S p(V)$ is a simply connected quasi-simple group with root system of type $C_{r}$ and the exterior powers $\wedge^{j} V$ have a good filtration as $\Omega(V)$-module. When $p>2$, for each $1 \leq i \leq r$ there is an exact sequence

$$
0 \rightarrow \bigwedge^{i-2} V \rightarrow \bigwedge^{i} V \rightarrow \nabla_{S p(V)}\left(\omega_{i}\right) \rightarrow 0
$$

of $\Omega(V)$-modules (where $\wedge^{-1} V=0$ and $\wedge^{0} V=k$ ).

Proof. It follows from [12], Theorem 25.11 that $\Omega(V)=S p(V)$ is quasisimple of type $C_{r}$. For $p>2$ the results on exterior powers of $V$ follow from [1] 4.9. The fact that the modules $\wedge^{j} V$ have a good filtration when $p=2$ follows from [8], Appendix A.

3.2.4. Assume that $\beta$ is symmetric and non-degenerate, that $p>2$ and that $\operatorname{dim} V=2 r+1$. Then

(a) $\Omega(V)=S O(V)$ is a quasisimple group of type $B_{r}$.

(b) $\mathfrak{\omega}_{i} \in X_{S O(V)}$ for $1 \leq i \leq r-1,2 \omega_{r} \in X_{S O(V)}$ and for any $\lambda \in X_{S O(V)}$, we have

$$
\lambda \in X_{S O(V)}^{+} \quad \text { if and only if } \quad \lambda \in \sum_{i=1}^{r-1} \mathbf{Z}_{\geq 0} \omega_{i}+\mathbf{Z}_{\geq 0} 2 \omega_{r}
$$

(c) For $1 \leq i \leq r-1, \nabla_{S O(V)}\left(\omega_{i}\right) \simeq \bigwedge^{i} V$, and $\nabla_{S O(V)}\left(2 \omega_{r}\right) \simeq \wedge^{r} V$.

Proof. (a) follows from [12], Theorem 25.10. Moreover, by loc. cit. one knows that $\Omega(V)$ is not simply connected. Now (b) follows from the description in [2], Plate II. Finally, (c) is verified in [1], 4.9.

3.2.5. Assume that $\beta$ is symmetric and non-degenerate, that $p>2$, and that $\operatorname{dim} V=2 r$. Then

(a) $\Omega(V)=S O(V)$ is a quasisimple group of type $D_{r}$.

(b) $\mathfrak{\omega}_{i} \in X_{S O(V)}$ for $1 \leq i \leq r-2, \omega_{r-1}+\omega_{r}, 2 \omega_{r-1}, 2 \omega_{r} \in X_{S O(V)}$ and for any $\lambda \in X_{S O(V)}$, we have

$$
\lambda \in X_{S O(V)}^{+} \quad \text { if and only if } \quad \lambda \in \sum_{i=1}^{r-2} \mathbf{Z}_{\geq 0} \omega_{i}+\mathbf{Z}_{\geq 0}\left(\omega_{r-1}+\omega_{r}\right)+\mathbf{Z}_{\geq 0} 2 \omega_{r-1}+\mathbf{Z}_{\geq 0} 2 \omega_{r} .
$$


(c) For $1 \leq i \leq r-2, \nabla_{S O(V)}\left(\omega_{i}\right) \simeq \bigwedge^{i} V$. Moreover,

$$
\bigwedge^{r-1} V \simeq \nabla_{S O(V)}\left(\omega_{r-1}+\omega_{r}\right) \quad \text { and } \quad \bigwedge^{r} V \simeq \nabla_{S O(V)}\left(2 \omega_{r}\right) \oplus \nabla_{S O(V)}\left(2 \omega_{r-1}\right)
$$

Proof. (a) follows from [12], Theorem 25.12. Moreover, by loc. cit. one knows that $\Omega(V)$ is neither simply connected nor adjoint. In this case, there are three groups in the isogeny class which are neither adjoint nor simply connected; $\Omega(V)$ is characterized by the fact that $X$ contains neither $\omega_{r-1}$ nor $\omega_{r}$.

Now (b) follows from the description in [2], Plate IV. The assertions in (c) about $\bigwedge^{i} V$ for $i<r$ are verified in [1], 4.9. The assertion about $\wedge^{r} V$ is proved in [16], Remark 3.4.

We conclude this discussion with the following result, which is similar to the methods used in [7] and [4].

3.2.6. Let $G$ be a classical group with natural representation $V$ and assume that $p>2$ if $G \neq S L(V)$. Let $H \subset G$ be a connected and reductive subgroup. Then $(G, H)$ is a Donkin pair if and only if the exterior algebra $\Lambda^{\bullet} V$ has a good filtration as an H-module.

Proof. Together with 2.2.1, the descriptions found in 3.2.2, 3.2.3, 3.2.4, and 3.2.5 show that the exterior algebra $\Lambda^{\bullet} V$ has a good filtration as $G$-module. Thus if $(G, H)$ is a Donkin pair then $\Lambda^{\bullet} V$ has a good filtration as $H$-module.

Conversely, suppose that $\Lambda^{\bullet} V$ has a good filtration as $H$-module. Of course, according to 2.2.1. also each exterior power $\bigwedge^{i} V$ has a good filtration as $H$-module.

To show that $(G, H)$ is a Donkin pair, we are going to apply 2.4.1. We verify for each classical group $G$ that there is a set of dominant weights $\lambda_{1}, \ldots, \lambda_{t} \in \mathcal{W}(G, H)$ for which $\lambda \in X$ satisfies

$$
(*) \quad \lambda \in X_{G}^{+} \quad \text { if and only if } \quad \lambda \in \sum_{i=1}^{t} \mathbf{Z}_{\geq 0} \lambda_{i} .
$$

When $G=S L(V)$, with $\operatorname{dim} V=r+1$, we take $t=r$ and we let $\lambda_{i}=\omega_{i}$ for $1 \leq i \leq r$. According to 3.2 .2

$$
\nabla_{S L(V)}\left(\lambda_{i}\right)=\nabla_{S L(V)}\left(\omega_{i}\right) \simeq \bigwedge^{i} V
$$

which has a good $H$-module filtration by assumption.

Now let $\beta$ be non-degenerate and alternating and let $\operatorname{dim} V=2 r$. Then 3.2.3 shows that $G=\Omega(V)$ is again simply connected; again we take $t=r$ and we let $\lambda_{i}=\omega_{i}$ for $1 \leq i \leq r$. Applying 3.2.3, we have for each $1 \leq i \leq r$ an exact sequence of $H$-modules

$$
0 \rightarrow \bigwedge^{i-2} V \rightarrow \bigwedge^{i} V \rightarrow \operatorname{res}_{H}^{\Omega(V)} \nabla_{\Omega(V)}\left(\omega_{i}\right) \rightarrow 0
$$

Applying 2.2.1 (a) together with our assumption, we conclude that $\operatorname{res}_{H}^{\Omega(V)} \nabla_{\Omega(V)}\left(\omega_{i}\right)$ has a good $H-$ module filtration for all $1 \leq i \leq r$.

Now let $\beta$ be symmetric and non-degenerate and let $\operatorname{dim} V=2 r+1$. We take $t=r$ and set $\lambda_{i}=\omega_{i}$ for $i<r$ and $\lambda_{r}=2 \omega_{r}$. Then 3.2.4 shows that the $\lambda_{i}$ satisfy $(*)$. Moreover, the same result shows that

$$
\operatorname{res}_{H}^{\Omega(V)} \nabla_{\Omega(V)}\left(\lambda_{i}\right) \simeq \bigwedge^{i} V
$$

has a good $H$-module filtration by assumption.

Finally, let $\beta$ be symmetric and non-degenerate and let $\operatorname{dim} V=2 r$. We take $t=r+1$, we set $\lambda_{i}=\omega_{i}$ for $i<r-1$, and we set $\lambda_{r-1}=\omega_{r}+\omega_{r+1}, \lambda_{r}=2 \omega_{r}$, and $\lambda_{r+1}=2 \omega_{r-1}$. It follows from 3.2.5 that $(*)$ holds. Now, the same result shows that the $H$-modules

$$
\operatorname{res}_{H}^{\Omega(V)} \nabla_{\Omega(V)}\left(\lambda_{i}\right) \simeq \bigwedge^{i} V \quad(1 \leq i \leq r-2), \quad \text { and } \quad \operatorname{res}_{H}^{\Omega(V)} \nabla_{\Omega(V)}\left(\lambda_{r-1}\right) \simeq \bigwedge^{r-1} V
$$


have a good $H$-filtration by assumption. Finally, since by 3.2 .5 we have

$$
\bigwedge^{r} V \simeq \operatorname{res}_{H}^{\Omega(V)} \nabla_{\Omega(V)}\left(2 \lambda_{r}\right) \oplus \operatorname{res}_{H}^{\Omega(V)} \nabla_{\Omega(V)}\left(2 \lambda_{r-1}\right)
$$

we conclude via 2.2.1 (b) that $\operatorname{res}_{H}^{\Omega(V)} \nabla_{\Omega(V)}\left(2 \lambda_{j}\right)$ has a good filtration as $H$-module for $j=r, r+1$. This completes the proof.

Recall from 3.2.2 and 3.2.3 that $S L(V)$ is simply connected, and $\Omega(V)$ is simply connected if $\beta$ is alternating. In order to study good filtration subgroups of the simply connected covers of orthogonal groups, one may use the following:

3.2.7. Suppose that $p>2$ and let $G=\Omega(V, \beta)$ for a non-degenerate symmetric form $\beta$. Let $\pi: G_{s c} \rightarrow G$ be the simply connected covering group of $G$, and let $H \subset G_{s c}$ be a connected and reductive subgroup. Then $\left(G_{s c}, H\right)$ is a Donkin pair if and only if $(G, \pi H)$ is a Donkin pair and one of the following conditions holds:

(a) $\operatorname{dim} V=2 r+1$ is odd and $\operatorname{res}_{H}^{G_{s c}} \nabla_{G_{s c}}\left(\omega_{r}\right)$ has a good filtration, with numbering of the fundamental dominant weights as in 3.2.4 or

(b) $\operatorname{dim} V=2 r$ is even and $\operatorname{res}_{H}^{G_{s c}} \nabla_{G_{s c}}\left(\omega_{i}\right)$ has a good filtration for $i=r-1, r$, with numbering of the fundamental dominant weights as in 3.2 .5

Proof. We apply 2.4.1 using the set of fundamental dominant weights as in Remark 2.4.2. The result now follows from the descriptions found in 3.2.4 and 3.2.5 together with 3.2.6.

\subsection{Some reductive subgroups of a classical group}

Let $V$ be a finite dimensional $k$-vector space, let $\beta$ be a non-degenerate alternating or symmetric bilinear form on $V$ and suppose that $p>2$. As above put $\Omega(V)=\Omega(V, \beta)$.

Let $W \subset V$ be a linear subspace of $V$. We say that $W$ is non-degenerate if the restriction of $\beta$ to $W$ is non-degenerate, and we say that $W$ is isotropic if the restriction of $\beta$ to $W$ is identically zero.

3.3.1. Let $W_{1}, \ldots, W_{r} \subset V$ be non-degenerate subspaces with $\beta\left(W_{i}, W_{j}\right)=0$ for $i \neq j$. Then

$$
\left(\Omega(V), \Omega\left(W_{1}\right) \times \cdots \times \Omega\left(W_{r}\right)\right)
$$

is a Donkin pair.

Proof. [4], Prop. 3.3; here we are using that $p>2$.

3.3.2. Let $P \subset \Omega(V)$ be a parabolic subgroup and let $L \subset P$ be a Levi factor. Then there is a non-degenerate subspace $W \subset V$ and an isotropic subspace $U \subset V$ such that $L \simeq L_{1} \times L_{2}$ where $L_{1}$ is equal to $\Omega(W)$ and $L_{2}$ is a Levi factor of a parabolic subgroup of $S L(U)$.

Proof. The result follows from the well-known observation that a parabolic subgroup of $\Omega$ is the stabilizer of a flag of isotropic subspaces of $V$.

\subsection{Some modules for $S L_{2}$ having a good filtration}

We write $S$ for the simple algebraic group $S L_{2}$.

Let $\omega \in X_{S}^{+}$denote the fundamental weight for some choice of maximal torus of Borel subgroup of $S$. For any integer $n \geq 0$ set $\nabla_{S}(n):=\nabla_{S}(n \omega)$, the unique induced $S$-module having dimension $n+1$.

3.4.1. The $S$-module $\nabla_{S}(n)$ is simple if and only if $n<p$.

Proof. This is a consequence of the Linkage Principle [11], §II.6.

A semisimple $S$-module $V$ will be called restricted (or restricted semisimple) if $\operatorname{dim}_{k} V^{S}=\operatorname{dim}_{k} V^{\operatorname{Lie}(S)}$. 
3.4.2. The following are equivalent for an $S$-module $V$.

(a) $V$ is a restricted semisimple $S$-module

(b) There is an isomorphism $V \simeq \bigoplus_{i \in I} \nabla_{S}\left(n_{i}\right)$ where $0 \leq n_{i}<p$ for all $i \in I$

(c) If $V_{m \omega} \neq 0$ then $m<p$.

Proof. First, suppose that $L$ is a simple $S$-module. If the highest weight $n \omega$ of $L$ satisfies $n<p$, it follows from [11], §II.3.15 that $L$ is simple as a module for $\operatorname{Lie}(S)$ as well. For any simple $S$-module $L$, it now follows from Steinberg's Tensor Product Theorem [11], II.3.17 that L is semisimple as a Lie(S)module. If $n \omega$ is the highest weight of $L$, Steinberg's Tensor Product Theorem yields $L^{S}=L^{\operatorname{Lie}(S)}$ if and only $n<p$. It is then clear that (a) implies (b). If (b) holds, evidently (c) holds as well.

Finally, if (c) holds, it follows from 3.4.1 that the simple submodules of $V$ all have the form $L(n)=$ $\nabla_{S}(n)$ for $n<p$. The Linkage Principle [11], II.6 shows that $\operatorname{Ext}_{S}^{1}(L(n), L(m))=0$ whenever $0 \leq$ $n, m<p$ so indeed $V$ is semisimple and (a) follows.

3.4.3. Let E be a finite dimensional restricted semisimple $S$-module. Then $\wedge^{\bullet} E$ has a good filtration as an S-module.

Proof. By hypothesis and 3.4.2, we may write $E=\bigoplus_{i=1}^{r} E_{i}$ where $E_{i} \simeq \nabla_{S}\left(n_{i}\right)$ with $0 \leq n_{i}<p$ $i=1, \ldots, r$. Then $\operatorname{dim} E_{i} \leq n_{i}+1 \leq p$ for each $i$.

We may evidently view $\wedge^{\bullet} E_{i}$ as an $S$-submodule of $\wedge^{\bullet} E$ for each $i$, and multiplication in the algebra $\Lambda^{\bullet} E$ defines a $S$-module isomorphism

$$
\bigwedge^{\bullet} E_{1} \otimes \bigwedge^{\bullet} E_{2} \otimes \cdots \otimes \bigwedge^{\bullet} E_{r} \stackrel{\sim}{\rightarrow} \bigwedge^{\bullet} E
$$

In view of 2.2.3, it suffices to prove the claim when $E=\nabla_{S}(n)$ for some $n<p$.

Now, $\wedge^{\bullet} E=\bigoplus_{i=0}^{n+1} \wedge^{i} E$ as $S$-modules, so it is enough to see that $\wedge^{i} E$ has a good filtration as $S$ module for each $0 \leq i \leq n+1$. Since $E$ has a good filtration as $S$-module, 2.2.5 shows that $\wedge^{i} E$ has a good filtration as $S$-module for each $i<p$. This completes the proof if $n<p-1$ since $\operatorname{dim} \nabla_{S}(n)=$ $n+1$. If $n=p-1$, it only remains to note that $\operatorname{dim} E=p$ so that $\Lambda^{p} E=k=\nabla_{S}(0)$ has a good filtration as $S$-module.

\subsection{Groups of exceptional type}

Let $G$ be a simply connected, quasisimple algebraic group whose root system $R$ is of exceptional type of rank $r$ : i.e. $R$ is one of $G_{2}, F_{4}, E_{6}, E_{7}$, or $E_{8}$. We always number the simple roots of $R$ according to the tables found in [2], Plate I-IX.

We begin with the following observations

3.5.1. Let $L$ be a Levi factor of a parabolic subgroup of $G$. Then $L$ is isomorphic to a direct product $L \simeq$ $L_{1} \times \cdots \times L_{t}$ where for each $1 \leq i \leq t$, one of the following holds:

(a) $L_{i}$ is a torus, or

(b) $L_{i}$ a simply connected quasisimple group of exceptional type, or

(c) $L_{i}$ is a simply connected quasisimple group of type $A_{r}$ with $r \leq 7$, or

(d) G has type $E_{d}$ for $d=6,7$, or 8 and $L_{i}$ is a simply connected quasisimple group of type $D_{r}$ with $r \leq d-1$, or

(e) G has type $F_{4}$ and $L_{i}$ is a simply connected quasisimple group of type $B_{2}=C_{2}, B_{3}$ or $C_{3}$.

Proof. Since $G$ is simply connected, $L$ is also simply connected, so that $L$ is isomorphic to the product of its connected center and its simply connected derived group $L^{\prime}$. The result now follows by inspection of the Dynkin diagram of $G$. 


\subsection{Good filtration subgroups of a group of exceptional type}

Let $W_{p}$ be the affine Weyl group associated with $G$ [11], $§ I I .6$. We consider the so-called "dot-action" of $W_{p}$ on the weight lattice $X=X^{*}(T)$ : for $w \in W_{p}$ and $\mu \in X$ we have $w \bullet \mu=w(\mu-\rho)+\rho$ where $\rho=\frac{1}{2} \sum_{\alpha>0} \alpha$ is the half-sum of positive roots.

For a reductive subgroup $H \subset G$, recall that $\mathcal{W}(G, H)$ denotes the set of dominant weights for $G$ for which the $H$-module $\operatorname{res}_{H}^{G} \nabla_{G}(\lambda)$ has a good filtration. Recall also that for a $G$-module $V$ with a good filtration, we write $\operatorname{Supp}(V)$ for the set of $\lambda \in X_{C}^{+}$for which $\nabla_{G}(\lambda)$ occurs as a layer in a good filtration of $V$. We first recall the following fact from [21].

3.6.1. Let $M$ be a G-module with good filtration for which $\operatorname{res}_{H}^{G} M$ has a good filtration as $H$-module. Let $\lambda \in \operatorname{Supp}(M)$. Suppose that one of the following holds for each $\mu \in \operatorname{Supp}(M)$ with $\mu \neq \lambda$ :

(a) $\mu<\lambda$ and $\mu \in \mathcal{W}(G, H)$, or

(b) $\mu \notin W_{p} \bullet \lambda$.

Then $\lambda \in \mathcal{W}(G, H)$.

Proof. This is Lemma 6.3, [21].

For any $G$-module $M$, let $\chi(M)$ denote the character of $M$; see [11], $§ I .2 .11$ and II.5. For $\lambda \in X_{G}^{+}$ set $\chi(\lambda):=\chi\left(\nabla_{G}(\lambda)\right)$.

3.6.2. The characters $\chi(\lambda)$ for $\lambda \in X_{G}^{+}$form a $\mathbf{Z}$-basis of $\mathbf{Z}[T]^{W}$. In particular, if the finite dimensional $G$ module $M$ has a good filtration, then $\chi(M)=\sum_{\lambda \in X_{G}^{+}} n_{\lambda} \chi(\lambda)$ where $n_{\lambda} \in \mathbf{Z}_{\geq 0}$ is equal to the number of layers in a good filtration of $M$ which are isomorphic to $\nabla_{G}(\lambda)$.

Proof. This follows from [11], Lemma and Remark II.5.8.

The following result may be viewed as a sharpened version of 2.4.1 valid for exceptional groups.

Theorem 3.6.3. Let $G$ be a simply connected quasisimple group of rank $r$ with exceptional type root system, and let $H \subset G$ be a reductive subgroup of $G$.

(i) If $G$ is of type $F_{4}, p \geq 5$, and $\omega_{4} \in \mathcal{W}(G, H)$, then $(G, H)$ is a Donkin pair.

(ii) If $G$ is of type $E_{6}, p \geq 5$, and $\omega_{1}, \omega_{6} \in \mathcal{W}(G, H)$, then $(G, H)$ is a Donkin pair.

(iii) If $G$ is of type $E_{7}, p \notin\{2,5,7\}$, and $\omega_{1}, \omega_{7} \in \mathcal{W}(G, H)$, then $(G, H)$ is a Donkin pair.

(iv) If $G$ is of type $E_{8}, p \geq 7$, and $\omega_{1}, \omega_{8} \in \mathcal{W}(G, H)$, then $(G, H)$ is a Donkin pair.

Proof. This theorem is proved using case-by-case computations with the computer program LiE [23]. Using 2.4.2, our goal is to show that $\omega_{i} \in \mathcal{W}(G, H)$ for $1 \leq i \leq r$. In each of the cases (i)-(iv), the hypothesis gives a set of fundamental weights known to be in $\mathcal{W}(G, H)$.

Next, using induced modules $\nabla_{G}(\lambda)$ for weights $\lambda$ known to be in $\mathcal{W}(G, H)$, we construct certain modules $F$ for which $F$ has a good filtration as $G$-module and $r e s_{H}^{G} F$ has a good filtration as $H$-module; that $F$ and $\operatorname{res}_{H}^{G} F$ have the required filtrations will in each case be clear from either 2.2.3 or 2.2.5. We then use the character $\chi(F)$ together with 3.6.2 to compute $\operatorname{Supp}(F)$; the computation of the character of $F$ is achieved in some cases using [23].

We now apply 3.6.1 to $F$ to obtain more weights known to lie in $\mathcal{W}(G, H)$ and then we repeat the procedure described above. The proof will be complete once we know that $\omega_{i} \in \mathcal{W}(G, H)$ for all $i$.

We describe details when $G$ has type $E_{7}$. In this case our initial assumption is that $\omega_{1}, \omega_{7} \in$ $\mathcal{W}(G, H)$. Recall we assume that $p \notin\{2,5,7\}$.

To argue that $\omega_{3} \in \mathcal{W}(G, H)$ we set $F=\wedge^{2} \nabla_{G}\left(\omega_{1}\right)$. Since $\chi(F)=\chi\left(\omega_{1}\right)+\chi\left(\omega_{3}\right)$, since $\omega_{1}<\omega_{3}$, and since $\omega_{1} \in \mathcal{W}(G, H)$, 3.6.1 shows that $\omega_{3} \in \mathcal{W}(G, H)$. 
Next, we set $F=\wedge^{2} \nabla_{G}\left(\omega_{7}\right)$. Since $\chi(F)=\chi(0)+\chi\left(\omega_{6}\right)$, 3.6.1 implies that $\omega_{6} \in \mathcal{W}(G, H)$.

Now set $F=\nabla_{G}\left(\omega_{1}\right) \otimes \nabla_{G}\left(\omega_{7}\right)$. The character of $F$ is given by $\chi(F)=\chi\left(\omega_{2}\right)+\chi\left(\omega_{7}\right)+\chi\left(\omega_{1}+\right.$ $\left.\omega_{7}\right)$. We have $\omega_{7}<\omega_{2}<\omega_{1}+\omega_{7}$, and $\omega_{2}$ is not in the $W_{p}$-orbit of $\omega_{1}+\omega_{7}$. Since $\omega_{1}, \omega_{7} \in \mathcal{W}(G, H)$, 3.6.1 implies that $\omega_{2} \in \mathcal{W}(G, H)$.

Also note that this implies $\omega_{1}+\omega_{7} \in \mathcal{W}(G, H)$, a fact we will use below when analyzing $\nabla_{G}\left(\omega_{1}\right) \otimes$ $\nabla_{G}\left(\omega_{2}\right)$.

The dominant weights $\mu$ for which $\mu \leq \omega_{2}+\omega_{7}$ are precisely $\omega_{2}+\omega_{7}, \omega_{1}, \omega_{3}, \omega_{6}$, and $2 \omega_{7}$. Of these, we already know $\omega_{1}, \omega_{3}, \omega_{6} \in \mathcal{W}(G, H)$. Setting $F=\operatorname{Sym}^{2} \nabla_{G}\left(\omega_{7}\right)$ it follows from 3.6.1 that $2 \omega_{7} \in \mathcal{W}(G, H)$. A similar argument shows that $2 \omega_{1} \in \mathcal{W}(G, H)$. Now set $F=\nabla_{G}\left(\omega_{2}\right) \otimes \nabla_{G}\left(\omega_{7}\right)$; for each dominant weight $\mu \neq \omega_{2}+\omega_{7}$ of $F$ we have $\mu \in \mathcal{W}(G, H)$ so that by 3.6.1 we have $\omega_{2}+\omega_{7} \in$ $\mathcal{W}(G, H)$.

Let $F=\Lambda^{2} \nabla_{G}\left(\omega_{2}\right)$ and observe that $\chi(F)=\chi(0)+\chi\left(\omega_{4}\right)+\chi\left(\omega_{6}\right)+\chi\left(\omega_{2}+\omega_{7}\right)+\chi\left(2 \omega_{1}\right)$. Let $\mu$ be a weight for which $\nabla_{G}(\mu)$ is isomorphic to a layer in a good filtration of $F$; then $\mu \leq \omega_{4}$. If $\mu \neq \omega_{4}$ we already know that $\mu \in \mathcal{W}(G, H)$. Thus by 3.6.1 we have $\omega_{4} \in \mathcal{W}(G, H)$.

Finally, set $F=\nabla_{G}\left(\omega_{1}\right) \otimes \nabla_{G}\left(\omega_{2}\right)$. Then $\chi(F)=\chi\left(\omega_{2}\right)+\chi\left(\omega_{5}\right)+\chi\left(\omega_{7}\right)+\chi\left(\omega_{1}+\omega_{7}\right)+\chi\left(\omega_{1}+\right.$ $\left.\omega_{2}\right)$. Thus, if $\mu$ is a weight for which $\nabla_{G}(\mu)$ is isomorphic to a layer of a good filtration of $F$, then $\mu \leq \omega_{5}$ if $\mu \neq \omega_{1}+\omega_{2}$. Now, $\omega_{1}+\omega_{2}$ is not in the $W_{p}$-orbit of $\omega_{5}$. Since $\omega_{2}, \omega_{7}, \omega_{1}+\omega_{7} \in \mathcal{W}(G, H)$, we conclude by 3.6.1 that $\omega_{5} \in \mathcal{W}(G, H)$, and we now have that $\omega_{i} \in \mathcal{W}(G, H)$ for all $1 \leq i \leq 7$. Hence the proof is completed for a group of type $E_{7}$.

Groups of type $F_{4}, E_{6}$ and $E_{8}$ are handled in a similar manner; we omit the details.

Remark 3.6.4. We used the following algorithm in LiE to determine, for two weights $\mu$ and $v$, the primes $p$ for which these two weights are conjugate under $W_{p}$.

Let $\Lambda_{R}$ denote the root lattice of $G$. First, we check if $\mu-v$ is in $\Lambda_{R}$; if not, the two weights are clearly not $W_{p}$-conjugate. Next, note that $\mu$ is $W_{p}$-conjugate to $v$ if and only if $w \bullet \mu-v \in p \Lambda_{R}$ for some $w \in W$, so we use LiE to compute the finite set

$$
\{w \bullet \mu-v: w \in W\}
$$

and determine for which primes $p$ the elements of this set are in $p \Lambda_{R}$.

\section{Main results}

\subsection{Optimal $S L_{2}$-subgroups of classical groups}

Let $G$ be a classical group with natural representation $V$ as in $\$ 3.1$ Recall that $p>2$ if $G \neq S L(V)$. Also recall the definition of optimal $S L_{2}$ subgroups from $\$ 2.5$

4.1.1. Let $\phi: S L_{2} \rightarrow G$ be a homomorphism of algebraic groups. Then $\phi$ is optimal if and only if $V$ affords a restricted semisimple module for $S L_{2}$.

Proof. This is observed in [18], Remark 18 when $G=S L(V)$, but the argument given there is valid for any $\Omega(V)$ as well.

Theorem 4.1.2. Let $S$ be an optimal $S L_{2}$-subgroup of the classical group $G$. Then $(G, S)$ is a Donkin pair.

Proof. Let $S \subset G$ be an optimal $S L_{2}$-subgroup, i.e. the image of an optimal homomorphism $S L_{2} \rightarrow G$. According to 4.1.1, the natural representation $V$ of $G$ is a restricted semisimple $S$-module. It now follows from 3.4 .3 that $\wedge^{\bullet} V$ has a good filtration as $S$-module. Finally, Theorem 3.2.6 now shows that $(G, S)$ is a Donkin pair, as required.

We now wish to investigate optimal $S L_{2}$-subgroups of the simply connected covering group $G_{s c}$ of the classical group $G$. Recall by 3.2.2 and 3.2.3 that the classical group $G$ is simply connected unless $G=\Omega(V, \beta)$ with $\beta$ a non-degenerate symmetric bilinear form. 
4.1.3. Let $\beta$ be a symmetric non-degenerate bilinear form on the finite dimensional vector space $V$, and let $S$ be an optimal $S L_{2}$-subgroup of $\Omega=\Omega(V, \beta)$. Then $S$ is distinguished if and only if res $\Omega_{S}^{\Omega} V$ is isomorphic as an $S$-module to a direct sum $\bigoplus_{i} V_{i}$ of simple $S$-submodules $V_{i} \simeq \nabla_{S}\left(n_{i}\right)$, where $n_{i}<p$ are even and pairwise distinct. If $S$ is distinguished, the restriction of $\beta$ to each $V_{i}$ is non-degenerate and $S \subset \prod_{i} \Omega\left(V_{i}\right) \subset \Omega$.

Proof. First suppose that $\operatorname{res}_{S}^{\Omega} V \simeq \bigoplus_{i} \nabla_{S}\left(n_{i}\right)$ with the $n_{i}$ as indicated. By 3.4.1 the $V_{i}=\nabla_{S}\left(n_{i}\right)$ are simple self-dual $S$-submodules of $V$ which are pairwise non-isomorphic, so the restriction of $\beta$ to $V_{i}$ is non-degenerate for each $i$. Thus $S$ is contained in the subgroup $\prod_{i} \Omega\left(V_{i}\right)$. Now, Schur's Lemma shows the endomorphism algebra $E n d_{S}(V)$ to be isomorphic to $\prod_{i} k$ with the $i$-th factor acting by scalar multiplication on $V_{i}$. Thus the centralizer $T=C_{G L(V)}(S)$ is the group of units $E_{n d}(V)^{\times} \simeq \prod_{i} \mathbf{G}_{m}$. In particular, the $G L(V)$-centralizer $T$ is contained in $\prod_{i} G L\left(V_{i}\right)$. But the intersection of $\prod_{i} G L\left(V_{i}\right)$ with $\Omega$ is precisely $\prod_{i} \Omega\left(V_{i}\right)$ and the intersection of $T$ with $\prod_{i} \Omega\left(V_{i}\right)$ is finite. Thus $C_{\Omega}(S)$ contains no positive dimensional torus and 2.5.4 implies that $S$ is distinguished.

For the converse, let $X \in \operatorname{Lie}(S)$ be nilpotent; since $S$ is assumed distinguished, the nilpotent element $X$ is distinguished. According to [10], Lemma 4.2 the partition describing the action of $X$ on $V$ has distinct odd parts. By 4.1.1, $V$ is a restricted semisimple $S$-module and the required description of the $S$-module res $\Omega_{S}^{\Omega} V$ now follows from 3.4.2.

Theorem 4.1.4. Let $\beta$ be a symmetric non-degenerate bilinear form on the finite dimensional vector space $V$, and write $G_{s c} \rightarrow \Omega(V, \beta)$ for the simply connected covering group of $\Omega(V, \beta)$. Write $d$ for the rank of $\Omega(V, \beta)$; thus $\operatorname{dim} V=2 d$ or $2 d+1$. Let $S \subset G_{s c}$ be an optimal $S L_{2}$-subgroup. Then $\left(G_{s c}, S\right)$ is a Donkin pair under the following assumptions:

(a) If $\operatorname{dim} V=2 d+1$ is odd, $p>\left(\begin{array}{c}d+1 \\ 2\end{array}\right)=\frac{(d+1) d}{2}$.

(b) If $\operatorname{dim} V=2 d$ is even, $p>\left(\begin{array}{l}d \\ 2\end{array}\right)=\frac{d(d-1)}{2}$.

Proof. Let $T$ be a maximal torus of the centralizer $C_{G_{s c}}(S)$; then $S$ is contained in the subgroup $M=$ $C_{G_{s c}}(T)$, and $M$ is a Levi factor of a parabolic subgroup of $G_{s c}$, hence $\left(G_{s c}, M\right)$ is a Donkin pair by 2.3.3. It now follows from 3.3.2 that $M \simeq M_{1} \times M_{2}$ where $M_{1}$ is the simply connected covering group of a Levi factor of a parabolic subgroup of some $S L(U)$ and $M_{2}$ is the simply connected covering group of $\Omega(W)$ for a non-degenerate subspace $W \subset V$.

To see that $S$ is a good filtration subgroup of $M$ one may use 2.3.5 thus, it is enough to see that $\left(M_{1}, S\right)$ and $\left(M_{2}, S\right)$ are Donkin pairs (where by abuse of notation we write $S$ for its image in $\left.M_{i}\right)$. Evidentally (the image of) $S$ is an optimal $S L_{2}$-subgroup of $M_{i}$ for $i=1,2$. Thus Theorem 4.1.2 already shows that $\left(M_{1}, S\right)$ is a Donkin pair.

It remains to argue that $\left(M_{2}, S\right)$ is a Donkin pair. Since $T$ is a maximal torus of $C_{G_{s c}}(S)$, evidentally $S$ is a distinguished optimal $S L_{2}$-subgroup of $M_{2}$. In particular, 4.1.3 shows that as an $S$-module, $W$ is isomorphic to a direct sum $\bigoplus_{i} V_{i}$ of simple $S$-submodules $V_{i} \simeq \nabla_{S}\left(n_{i}\right)$ where $n_{i}<p$ are even and pairwise distinct. Moreover, $S$ acts on $W$ through its image in $\prod_{i} \Omega\left(V_{i}\right)$, hence $S$ is contained in the product $\prod_{i} \Omega\left(V_{i}\right)_{s c} \subset M_{2}$. According to 2.3.5 to see that $\left(M_{2}, S\right)$ is a Donkin pair, it suffices to see that the image of $S$ in $\Omega\left(V_{i}\right)_{s c}$ is a good filtration subgroup for each $i$. Thus we may and will suppose that $M_{2}=\Omega(W)_{s c}$ is the simply connected cover of $\Omega(W)$ with $\operatorname{dim} W=2 d^{\prime}+1$ odd for some $d^{\prime} \leq d$, and that $S$ acts irreducibly on $W$.

Using Theorem 4.1.2 together with 3.2.7, we see that $S$ will be a good filtration subgroup of $M_{2}$ provided that the spin module $L=\nabla_{\Omega(W)_{s c}}\left(\omega_{d^{\prime}}\right)$ has a good filtration as $S$-module. Since $\omega_{d^{\prime}}$ is a minscule weight for the root system $B_{d^{\prime}}$, one knows that the weights of the spin representation are precisely the Weyl group conjugates of $\omega_{d^{\prime}}$. Using this description, one sees that when viewed as a module for $S$, the highest weight of the spin module $L$ is

$$
\frac{1}{2}\left(\sum_{i=1}^{d^{\prime}} 2 i\right)=\left(\begin{array}{c}
d^{\prime}+1 \\
2
\end{array}\right) \text {. }
$$


Thus if $p>\left(\begin{array}{c}d^{\prime}+1 \\ 2\end{array}\right)$, it follows from 3.4.2 that $\operatorname{res}_{S}^{\Omega(W)_{s c}}(L)$ is a restricted semisimple $S$-module, and hence has a good $S$-filtration.

Now, using the fact that $d^{\prime} \leq d$ and that $d^{\prime}<d$ if $\operatorname{dim} V$ is even (since in this case $2 d^{\prime}+1=$ $\operatorname{dim} W \leq \operatorname{dim} V=2 d)$, the conditions on $p$ and $d$ we have given in the statement suffice to guarantee that $\left(G_{s c}, S\right)$ is a Donkin pair.

Remark 4.1.5. Let $\beta$ be a non-degenerate symmetric bilinear form on $V$, and let $\Omega_{s c}$ be the simply connected covering group of $\Omega(V, \beta)$. The preceding theorem shows that any optimal $S L_{2}$-subgroup $S \subset \Omega_{s c}$ is a good filtration subgroup under the conditions indicated in the following tables:

\begin{tabular}{|c|c|c|}
\hline root system of $\Omega(V)$ & $\operatorname{dim} V$ & condition \\
\hline$D_{3}$ & 6 & $p \geq 5$ \\
$D_{4}$ & 8 & $p \geq 7$ \\
$D_{5}$ & 10 & $p \geq 11$ \\
$D_{6}$ & 12 & $p \geq 17$ \\
$D_{7}$ & 14 & $p \geq 23$ \\
\hline
\end{tabular}

\begin{tabular}{|c|c|c|}
\hline root system of $\Omega(V)$ & $\operatorname{dim} V$ & condition \\
\hline$B_{2}$ & 5 & $p \geq 7$ \\
$B_{3}$ & 7 & $p \geq 11$ \\
\hline
\end{tabular}

\subsection{Optimal $S L_{2}$-subgroups of exceptional type groups}

We now turn our attention to the exceptional groups.

Theorem 4.2.1. Let $G$ be a quasisimple group of exceptional type and let $S \subset G$ be an optimal $S L_{2}$-subgroup. Assume that $p$ is a good prime for $G$. If $S$ is distinguished then $(G, S)$ is a Donkin pair. More generally, if $S$ is an arbitrary optimal $S L_{2}$ subgroup then $(G, S)$ is a Donkin pair under the following additional conditions on p:

- If $G$ is of type $E_{6}$ or $F_{4}$ then $p \geq 11$;

- If $G$ is of type $E_{7}$ then $p \geq 17$;

- If $G$ is of type $E_{8}$ then $p \geq 23$.

Proof. Assume that $p$ is a good prime for $G$. We first prove, using a series of case-by-case computation of branching rules using the computer program $\operatorname{LiE}[23]$, that $(G, S)$ is a Donkin pair for every distinguished optimal $S L_{2}$ subgroup $S$ of a simply-connected quasisimple group $G$ of exceptional type (see below for an example). The result for non-simply-connected $G$ then follows from 2.3.6.

Now assume that $S$ is a non-distinguished optimal $S L_{2}$ subgroup of $G$ and assume the additional conditions given for $p$. We start by following the technique of the proof of Theorem 4.1.4 to reduce to the distinguished case. Let $T$ be a maximal torus of $C_{G}(S)$. Then $M=C_{G}(T)$ is a Levi factor of a parabolic subgroup of $G$ and $S$ is a distinguished optimal $S L_{2}$ subgroup of $M$. According to 2.3.3. $(G, M)$ is a Donkin pair; thus to prove the Theorem it suffices to prove that $(M, S)$ is a Donkin pair.

Now, since $S$ is assumed to not be distinguished in $G$, the derived group of $M$ has semisimple rank less than that of $G$. Hence we can proceed by induction on the rank of $M$. The case where rank $M=1$ is trivial. By 2.3.5 we may assume that $M$ is quasisimple. As we already know that $(M, S)$ is a Donkin pair when $M$ is of exceptional type (since $S$ is distinguished in $M$ ) we may assume that $M$ is not of exceptional type. Since $M$ is the Levi factor of a parabolic subgroup of an exceptional group, 3.5.1tells us the possibilities for the type of $M$. The induction step, and hence the result, now follows from Theorem 4.1.2. Theorem 4.1.4, and Remark4.1.5

We conclude the proof with an illustrative example of the use of $\mathrm{LiE}$ for the case where $G$ is of type $E_{7}$ and $S$ is distinguished. For any $S$-module $M$, let $\chi_{S}(M)$ denote the character of $M$ as an $S$-module; we also write $\chi_{S}(M)=\chi_{S}\left(\operatorname{res}_{S}^{G} M\right)$ for a $G$-module $M$. For any integer $n$ set $\chi_{S}(n):=\chi_{S}\left(\nabla_{S}(n \omega)\right)$, where $\omega$ denotes the single fundamental weight of $S$.

Assume that $G$ is simply-connected of type $E_{7}$. We use the tables starting on p. 175 of [5] (and the labels therein) to check each distinguished $S L_{2}$ of $G$. For each distinguished type $S$ we verify that the 
required modules for $G$ given in Theorem 3.6.3 afford restricted semisimple modules for $S$ and are hence good filtration modules for $S$. To do this, we check that the $S$-characters of these modules are sums of characters of simple induced modules $\nabla_{S}(n)$ for $n<p$ (cf 3.4.1 and 3.4.2).

We also need to determine the primes $p$ for which there is an optimal $S L_{2}$ morphism $\phi: S L_{2} \rightarrow$ $G$ corresponding to the given distinguished class. Let $X \in \operatorname{Lie}(B)$ be a nilpotent element in the distinguished orbit; then, by Theorem 2.5.3, it suffices to verify that $X^{[p]}=0$. Let $\mathfrak{g}=\oplus \mathfrak{g}(i)$ be the decomposition of $\mathfrak{g}:=\operatorname{Lie}(G)$ coming from the cocharacter associated to $X$; then by Proposition 24 in [15], $X^{[p]}=0$ if and only if $\mathfrak{g}(i)=0$ for all $i \geq 2 p$. We use this criterion to determine for which primes $X^{[p]}=0$.

- When $\operatorname{Lie}(S)$ contains a regular nilpotent element of $G$, we have $\chi_{S}\left(\nabla_{G}\left(\omega_{1}\right)\right)=35 \chi_{S}(0)+$ $31 \chi_{S}(2)+\chi_{S}(4)$ and $\chi_{S}\left(\nabla_{G}\left(\omega_{7}\right)\right)=\chi_{S}(0)+12 \chi_{S}(2)$. Furthermore, there is an optimal $S L_{2}$ homomorphism corresponding to the regular nilpotent orbit when $p \geq 17$.

- When $S$ is of type $E_{7}\left(a_{1}\right)$ or $E_{7}\left(a_{5}\right)$ we have $\chi_{S}\left(\nabla_{G}\left(\omega_{1}\right)\right)=17 \chi_{S}(0)+22 \chi_{S}(2)+10 \chi_{S}(4)$ and $\chi_{S}\left(\nabla_{G}\left(\omega_{7}\right)\right)=4 \chi_{S}(0)+14 \chi_{S}(2)+2 \chi_{S}(4)$. For type $E_{7}\left(a_{1}\right)$ there is an optimal $S L_{2}$ homomorphism corresponding to this distinguished type when $p \geq 13$ and for type $E_{7}\left(a_{5}\right)$ we need $p \geq 5$.

- When $S$ is of type $E_{7}\left(a_{2}\right), E_{7}\left(a_{3}\right)$, or $E_{7}\left(a_{4}\right)$ we have $\chi_{S}\left(\nabla_{G}\left(\omega_{1}\right)\right)=9 \chi_{S}(0)+15 \chi_{S}(2)+13 \chi_{S}(4)+$ $2 \chi_{S}(6)$ and $\chi_{S}\left(\nabla_{G}\left(\omega_{7}\right)\right)=8 \chi_{S}(0)+6 \chi_{S}(2)+6 \chi_{S}(4)$. For types $E_{7}\left(a_{2}\right)$ and $E_{7}\left(a_{3}\right)$ there is an optimal $S L_{2}$ homomorphism corresponding to this distinguished type when $p \geq 11$; for type $E_{7}\left(a_{4}\right)$ we need $p \geq 7$.

\subsection{Subsystem subgroups in the exceptional cases}

Let $G$ be a semisimple group. Recall that a subsystem subgroup of a semisimple group $G$ is a connected semisimple subgroup which is normalized by a maximal torus of $G$. In this section we consider pairs $(G, H)$ where $H$ is a subsystem subgroup of $G$. Remark that Levi factors of parabolic subgroups of $G$ are subsystem subgroups; these Levi factors are good filtration subgroups by 2.3 .3

Remark 4.3.1 (cf [13]). Subsystem subgroups of $G$ can be characterized as follows. Recall that $R$ is the root system of $G$. For any sub-root system $R^{\prime} \subseteq R$ let $H$ be the subgroup of $G$ generated by $T$ and the root subgroups $\left\{U_{\beta}: \beta \in R^{\prime}\right\}$. Then $H$ is a subsystem subgroup of $G$ and every subsystem subgroup of $G$ can be obtained in this way.

There is also a nice inductive procedure for constructing all subsystem subgroups of G. Starting with the extended Dynkin diagram of $G$, remove any collection of nodes. This gives the Dynkin diagram $D^{\prime}$ of a subsystem subgroup, and there is one conjugacy class of subsystem subgroups for each subdiagram obtained in this way. Now repeat this process with the connected components of the subdiagram $D^{\prime}$. In this fashion one obtains all subsystem subgroups of $G$. In particular, there are only finitely many conjugacy classes of subsystem subgroups.

A subsystem subgroup of maximal rank (= rank $G$ ) is the centralizer of an involution if and only if it is obtained via the technique of Remark 4.3.1 by removing a node from the extended Dynkin diagram with label 2 (where we label the extended Dynkin diagram based on the coefficients occurring in the highest root). We first recall the following theorem.

Theorem 4.3.2 ([4], Proposition 3.3 and [21], §6). Let $H$ be a reductive subgroup of $G$ which is the centralizer of an involution. If not all components of $G$ are of type $A_{r}$ or $C_{r}$ assume that $p>2$. Then $(G, H)$ is a Donkin pair. In particular, if $G$ has no components of exceptional type then $(G, H)$ is a Donkin pair for every subsystem subgroup $H$ of $G$. 
Recall that if we have reductive subgroups $H \subseteq H^{\prime} \subseteq G$ such that $\left(G, H^{\prime}\right)$ and $\left(H^{\prime}, H\right)$ are Donkin pairs, then also $(G, H)$ is a Donkin pair. Thus, to determine for which primes a given subsystem subgroup $H \subset G$ will be a good filtration subgroup, it suffices to consider the case where $H$ has maximal rank (= rank $G$ ). In the following theorem we consider the remaining subsystem subgroups of maximal rank which are not covered by Theorem 4.3.2. This list can be obtained by the method of Remark 4.3.1 above.

Theorem 4.3.3. Suppose that $p>2$, let $G$ be a quasisimple group of exceptional type and let $H \subset G$ be a subsystem subgroup of maximal rank such that $H$ is not the centralizer of an involution. Then the pair $(G, H)$ appears in the following list, and $H$ is a good filtration subgroup of $G$ provided that $p$ satisfies the indicated condition.

- $G$ is simply-connected of type $F_{4}$

$$
\begin{aligned}
& \circ A_{2} \times A_{2}: p \geq 5 . \\
& \circ A_{3} \times A_{1}: p \geq 5 .
\end{aligned}
$$

- $G$ is simply-connected of type $G_{2}$

$$
\text { ○ } A_{2}: p \neq 3 \text {. }
$$

- $G$ is simply-connected of type $E_{6}$

$$
\text { ○ } A_{2} \times A_{2} \times A_{2}: p \geq 5 \text {. }
$$

- $G$ is simply-connected of type $E_{7}$

○ $A_{5} \times A_{2}: p \notin\{2,5,7\}$.

- $G$ is simply-connected of type $E_{8}$

$$
\begin{aligned}
& \circ A_{8}: p \geq 7 \\
& \circ A_{1} \times A_{2} \times A_{5}: p \geq 7 \\
& \circ A_{4} \times A_{4}: p \geq 7 \\
& \circ D_{5} \times A_{3}: p \geq 7 \\
& \circ E_{6} \times A_{2}: p \geq 7 \\
& \circ A_{1} \times A_{7}: p \geq 11 .
\end{aligned}
$$

Proof. As with Theorem 4.2.1, this theorem is proved by a case-by-case computation using branching rules in $\mathrm{LiE}$. For the given group $G$ of exceptional type and subsystem subgroup $H$ we first use $\mathrm{LiE}$ to compute the characters of the appropriate modules from Theorem 3.6 .3 considered as $H$-modules. This character is written as a sum of characters of induced modules for $H$. We then find the minimal prime $p^{\prime}$ such that the highest weights of these induced modules for $H$ lie in the low alcove. This implies that for $p \geq p^{\prime}$ the $G$-modules from Theorem 3.6.3 afford simple induced modules for $H$ and thus are good filtration modules for $H$. Hence that theorem implies that $(G, H)$ is a Donkin pair for $p \geq p^{\prime}$. In addition, we can sometimes extend our analysis using linkage, as indicated in the example below.

As in the proof of Theorem 4.2.1, we will not give the details of each computation; we will instead give one illustrative example.

Let $G$ be simply-connected of type $E_{8}$ and let $H \subset G$ be the subsystem subgroup of type $D_{5} \times$ $A_{3}$. For a dominant weight $\mu$ of $H$ let $\chi_{H}(\mu)$ denote the character of the induced module $\nabla_{H}(\mu)$ with highest weight $\mu$. We write the weights of $D_{5} \times A_{3}$ as $(a, b, c, d, e, f, g, h)$, where $(a, b, c, d, e)$ is 
considered as a weight of $D_{5}$ and $(f, g, h)$ is considered as a weight of $A_{3}$. Then the character of $\nabla_{G}\left(\omega_{1}\right)$ as an $H$-module is $\sum_{\mu \in C} \chi_{H}(\mu)$, where $C$ is the following set of dominant weights for $H$ :

$$
\begin{aligned}
& \{(0),(0,0,0,0,0,0,2,0),(0,0,0,0,0,1,0,1),(0,0,0,0,1,0,1,1) \\
& (0,0,0,0,1,1,0,0),(0,0,0,1,0,0,0,1),(0,0,0,1,0,1,1,0),(0,0,0,1,1,0,0,0) \\
& (0,0,1,0,0,0,1,0),(0,1,0,0,0,1,0,1)(1,0,0,0,0,0,0,2),(1,0,0,0,0,0,1,0) \\
& (1,0,0,0,0,2,0,0),(1,0,0,0,1,0,0,1),(1,0,0,1,0,1,0,0),(2,0,0,0,0,0,0,0)\}
\end{aligned}
$$

Also, the character of $\nabla_{G}\left(\omega_{8}\right)$ as an $H$-module is $\sum_{\mu \in C^{\prime}} \chi_{H}(\mu)$, where $C^{\prime}$ is the following set of dominant weights for $H$ :

$$
\{(0,0,0,0,0,1,0,1),(0,0,0,0,1,1,0,0),(0,0,0,1,0,0,0,1),(0,1,0,0,0,0,0,0),(1,0,0,0,0,0,1,0)\} .
$$

We now consider the weights in $C \cup C^{\prime}$ to find primes for which the associated induced modules for $H$ are all simple. For example, consider the weight $(0,0,0,1,0,1,1,0) \in C$. The induced module with highest weight $(0,0,0,1,0)$ for $D_{5}$ is in the low alcove for $D_{5}$ when $p \geq 11$ and the induced module with highest weight $(1,1,0)$ for $A_{3}$ is in the low alcove for $A_{3}$ when $p \geq 5$. Thus $\nabla_{H}(0,0,0,1,0,1,1,0)$ is simple when $p \geq 11$.

Checking all the above weights in this manner, we see that for all $\mu \in C \cup C^{\prime}, \chi_{H}(\mu)$ is the character of a simple $H$-module for $p \geq 11$.

To further extend the analysis we consider linkage. We check to see if we can find a prime $<11$ so that $\mu$ is the minimal dominant weight in its linkage class for all $\mu \in C \cup C^{\prime}$; if so, $\chi_{H}(\mu)$ will be the character of a simple $H$-module for that prime also. This linkage computation now shows that for $p=7, \chi_{H}(\mu)$ is the character of a simple induced module for all $\mu \in C \cup C^{\prime}$. Thus $H$ is a good filtration subgroup of $G$ for $p \geq 7$.

Remark 4.3.4. In Theorem 4.3.3, the primes listed give sufficient but not, a priori, necessary conditions for the given subsystem subgroup to be a good filtration subgroup. One could perhaps extend the results in the theorem to more primes using a finer analysis of alcove considerations and linkage or by using 2.4.1 rather than Theorem 3.6.3

\section{References}

[1] Henning Haahr Andersen and Jens Carsten Jantzen, Cohomology of induced representations for algebraic groups, Math. Ann. 269, no. 4, 487-525.

[2] Nicolas Bourbaki, Lie groups and Lie algebras. Chapters 4-6, Elements of Mathematics (Berlin), Springer-Verlag, Translated from the 1968 French original by Andrew Pressley.

[3] Michel Brion and Shrawan Kumar, Frobenius splitting methods in geometry and representation theory, Progress in Mathematics, no. 231, Birkhäuser Boston, 2005.

[4] Jonathan Brundan, Dense orbits and double cosets, NATO Adv. Sci. Inst. Ser. C Math. Phys. Sci., vol. 517, pp. 259-274, Kluwer Acad. Publ.

[5] Roger W. Carter, Finite groups of Lie type, Wiley Classics Library, John Wiley \& Sons Ltd., Conjugacy classes and complex characters; Reprint of the 1985 original; A Wiley-Interscience Publication.

[6] Stephen Donkin, The normality of closures of conjugacy classes of matrices, Invent. Math. 101, no. 3, 717-736. 
[7] _ Rational representations of algebraic groups, Lecture Notes in Mathematics, vol. 1140, Springer-Verlag, Tensor products and filtration.

[8] _ On tilting modules and invariants for algebraic groups, Finite-dimensional algebras and related topics (Ottawa, ON, 1992), NATO Adv. Sci. Inst. Ser. C Math. Phys. Sci., vol. 424, Kluwer Acad. Publ., Dordrecht, 1994, pp. 59-77.

[9] James E. Humphreys, Introduction to Lie algebras and representation theory, Graduate Texts in Mathematics, vol. 9, Springer-Verlag, Second printing, revised.

[10] Jens Carsten Jantzen, Nilpotent orbits in representation theory, Lie Theory: Lie Algebras and Representations (J-P Anker, ed.), Progress in Mathematics, vol. 228, Birkhäuser, pp. 1-211.

[11] _ Representations of algebraic groups, 2 ed., Mathematical Surveys and Monographs, vol. 107, American Mathematical Society.

[12] Max-Albert Knus, Alexander Merkurjev, Markus Rost, and Jean-Pierre Tignol, The book of involutions, American Mathematical Society Colloquium Publications, vol. 44, American Mathematical Society.

[13] Martin W. Liebeck, Introduction to the subgroup structure of algebraic groups, Representations of reductive groups, Publ. Newton Inst., Cambridge Univ. Press, Cambridge, 1998, pp. 129-149.

[14] Olivier Mathieu, Filtrations of G-modules, Ann. Sci. École Norm. Sup. (4) 23, no. 4, 625-644.

[15] George J. McNinch, Optimal SL(2)-homomorphisms, Comment. Math. Helv. 80, 391 - 426.

[16] _ Semisimplicity of exterior powers of semisimple representations of groups, J. Algebra 225, no. 2, 646-666.

[17] _ Nilpotent orbits over ground fields of good characteristic, Math. Annalen 329 (2004), 49 - 85, arXiv:math.RT/0209151.

[18] George J. McNinch and Donna M. Testerman, Completely reducible SL(2)-homomorphisms, Transactions of the AMS 359, arXiv:math.RT/0510377.

[19] Alexander Premet, Nilpotent orbits in good characteristic and the Kempf-Rousseau theory, J. Alg 260, $338-366$.

[20] Gary M. Seitz, Unipotent elements, tilting modules, and saturation, Invent. Math. 141, no. 3, 467-502.

[21] Wilberd van der Kallen, Steinberg modules and Donkin pairs, Transform. Groups 6, no. 1, 87-98.

[22] __ Lectures on Frobenius splittings and B-modules, Published for the Tata Institute of Fundamental Research, Bombay, 1993.

[23] Marc van Leeuwen, Arjeh Cohen, and Bert Lisser, Lie : A package for Lie group computations. 